\title{
Periodismo transmedia y consumo mediático de la generación millennials ${ }^{*}$
}

\author{
Kelly Robledo-Dioses ${ }^{* *}$ \\ Tomás Atarama-Rojas ***
}

Recibido: 2017-10-2. Enviado a pares: 2017-10-28.

Aprobado por pares: 2017-11-28. Aceptado: 2018-01-05

https://doi.org/10.22395/angr.v17n33a5

\section{Resumen}

El escenario mediático actual, marcado por cambios tecnológicos y narrativos, exige a los periodistas una constante actualización para mantener la conexión con su público. Este artículo presenta al transmedia storytelling como el mejor aliado del periodismo para la difusión de hechos. Este modelo comunicativo otorga una naturaleza de expansión, multiplicidad y profundidad a las historias, que permite elevar el nivel de participación del público en cada medio en el que desarrolle la información.

En concreto, la investigación busca detectar los modos en los que el periodismo se puede valer de la narrativa transmedia para alcanzar y generar interacción en la generación millennial. Para lograr este objetivo, se ha partido de un estudio mayor que analiza el consumo de medios que hacen los jóvenes de la región de Piura, en Perú, en específico, alumnos de $5^{\circ}$ de secundaria de los colegios privados de la zona.

Gracias al estudio, se encontró que los estudiantes de Piura realizan un consumo casi masivo de los medios digitales, en especial, de las redes sociales. Además, como puerta de entrada a los contenidos ofrecidos en estas plataformas, recurren en gran medida a los dispositivos móviles. Se entiende así que las historias periodísticas deben estar presentes en estos medios con contenidos atractivos en fondo y forma que respondan a satisfacer a este perfil de lector/espectador/usuario millennial que valora mucho el contenido de calidad.

Palabras clave: periodismo, narrativa transmedia, generación millennial, consumo de medios, medios digitales, estudiantes, redes sociales, dispositivos móviles.

Este producto ve su origen en una investigación más amplia denominada Los escolares piuranos en la era digital: un estudio a partir de la experiencia de los alumnos de $5^{\circ}$ de secundaria de los colegios privados de la región (2016), un trabajo financiado por el Centro de Investigación en Opinión Pública de la Universidad de Piura, Perú.

. Kelly Robledo-Dioses es licenciada en Comunicación por la Universidad de Piura (Perú). Actualmente se desempeña como profesora investigadora de la Facultad de Comunicación de la Universidad de Piura (Perú) donde imparte las asignaturas Comunicación Escrita 1, Géneros y Estilos Informativos e Investigación en Comunicación 1 y 2. Su investigación se centra en el periodismo, la comunicación transmedia y el storytelling corporativo. kelly.robledo@ udep.pe Orcid: https://orcid.org/0000-0003-1409-3773

... Tomás Atarama-Rojas es máster en Creación de Guiones Audiovisuales por la Universidad Internacional de La Rioja (España) y licenciado en Comunicación por la Universidad de Piura (Perú). Actualmente se desempeña como profesor investigador de la Facultad de Comunicación de la Universidad de Piura (Perú), donde imparte las asignaturas Comunicación Narrativa y Storytelling y Fundamentos de Guion. Su investigación está centrada en el guion cinematográfico y el uso del storytelling en el mundo comercial y corporativo. tomas.atarama@udep.pe Orcid: https://orcid. org/0000-0002-4430-3391 


\title{
Transmedia journalism and media consumption of the millennial generation
}

\begin{abstract}
The current media scenario, marked by technological and narrative changes, requires journalists to constantly bring themselves up to date in order to maintain the connection with their audience. This article presents transmedia storytelling as the best ally of journalism for the dissemination of facts. This communicative model grants a nature of expansion, multiplicity and depth to the stories, which allows to raise the level of participation of the public in each medium in which information is developed.

Specifically, this research seeks to detect the ways in which journalism can use transmedia narrative to reach and generate interaction in the millennial generation. To achieve this goal, a larger study that analyzes the consumption of resources made by young people in the region of Piura, in Peru, specifically, students in 5th year of secondary school in private schools in the area, was taken as a starting point.

Thanks to the study, it was found that Piura students consume high, almost massive, amounts of digital media, especially social networks. In addition, they rely heavily on mobile devices as a gateway to the content offered on these platforms. Thus, news stories must be present in these media with attractive content in substance and form, that can satisfy this reader / viewer / millennial user who highly values quality content.
\end{abstract}

Keywords: journalism, transmedia narrative, millennial generation, media consumption, digital media, students, social networks, mobile devices.

\section{Jornalismo transmídia e consumo midiático da geração millennials}

\begin{abstract}
Resumo
O cenário midiático atual, marcado por mudanças tecnológicas e narrativas, exige dos jornalistas uma constante atualização para manter a conexão com seu público. Este artigo apresenta o transmídia storytelling como o melhor aliado do jornalismo para a difusão de fatos. Esse modelo comunicativo outorga uma natureza de expansão, multiplicidade e profundidade às histórias, que permite elevar o nível de participação do público em cada meio no qual desenvolva a informação.

Mais especificamente, a pesquisa procura detectar os meios nos quais o jornalismo pode usar a narrativa transmídia para atingir e gerar interação na geração millennial. Para alcançar esse objetivo, partiu-se de um estudo maior que analisa o consumo de meios que fazem os jovens da região de Piura, no Peru, em específico, alunos do $5^{\circ}$ ano do Ensino Fundamental dos colégios privados da área.

Graças ao estudo, percebeu-se que os estudantes de Piura realizam um consumo quase em massa dos meios digitais, em especial, das redes sociais. Além disso, como porta de entrada aos conteúdos oferecidos nessas plataformas, recorrem, em grande parte, aos dispositivos móveis. Entende-se, desse modo, que as histórias jornalísticas devem estar presentes nesses meios com assuntos atraentes no conteúdo e na forma e que tentem satisfazer esse perfil de leitor/espectador/usuário millennial que valoriza muito o conteúdo de qualidade.
\end{abstract}

Palavras-chave: jornalismo, narrativa transmídia, geração millennial, consumo de meios, meios digitais, estudantes, redes sociais, dispositivos móveis. 


\section{Introducción}

"Sobrevivir en entornos informativos que se trasforman a gran velocidad exige a los medios desarrollar la capacidad para aprender rápido y adquirir las destrezas necesarias para implementar los cambios con eficacia" (Orihuela, 2011, p. 25). Nos encontramos en un escenario donde la aparición de diversos medios ha generado el nacimiento de nuevos modelos de comunicación que buscan el uso conjunto de los distintos canales para la difusión de contenidos (Robledo, Atarama y Palomino, 2017).

Este contexto potencia la implementación de la narrativa transmedia, modelo comunicativo en el que una historia global "se desarrolla a través de múltiples plataformas mediáticas, y cada nuevo texto hace una contribución específica y valiosa a la totalidad" (Jenkins, 2008, p. 101). El modelo transmedia otorga una naturaleza de expansión, multiplicidad y profundidad a las historias que eleva el nivel de participación del público en cada medio por el que discurre el hecho noticioso (Robledo et al., 2017).

La presente investigación explora cómo el periodismo se puede favorecer de las herramientas transmedia para llegar a un público joven, como el millennial. Para encontrar los puntos de contacto y las posibilidades narrativas, se presentan datos empíricos sobre el consumo de medios de los alumnos de $5^{\circ}$ de secundaria de Piura, Perú. Esta información ha sido extraída de una investigación más amplia realizada por el Centro de Investigación en Opinión Pública de la Universidad de Piura, denominada: Los escolares piuranos en la era digital: un estudio a partir de la experiencia de los alumnos de $5^{\circ}$ de secundaria de los colegios privados de la región (2016).

Se tiene como objetivo conocer los hábitos de consumo de medios para así detectar los modos en los que el periodismo transmedia puede alcanzar y generar interacción con este público, parte de la generación millennial, una audiencia con características muy particulares que se constituye en un reto para el periodismo.

\section{El escenario transmedia: un desafío para el periodismo}

Kovach y Rosenstiel (2012, p. 24) señalan que "el propósito principal del periodismo es proporcionar a los ciudadanos la información que necesitan para ser libres y capaces de gobernarse a sí mismos". De esta forma, "el buen periodista (...) es capaz de orientar al lector en sus dudas y llenar sus vacíos de información, desde los más domésticos hasta los más decisivos para él como individuo y para la sociedad a la que pertenece" (Darío y Astudillo, 2005, p. 101).

Se entiende así que el profesional del periodismo necesita comunicar a su audiencia aquella información que se supone relevante, pero, ¿̇cómo dar continuidad a esta labor periodística en el contexto actual? Estamos en un escenario en el que, por un lado, la existencia de nuevos fenómenos de comunicación, fruto de la interacción de los diseños comunicativos y la innovación tecnológica, amplía las posibilidades comunicativas huma- 
nas, y, por otro lado, las empresas periodísticas deben repensar sus modelos de gestión y producción, pero también sus tareas y sus contenidos (Arrojo, 2015).

La lógica de distribución de contenidos periodísticos se ha visto potenciada, de manera que "lo que tradicionalmente había sido una difusión compartimentada del mensaje informativo, dividida entre distintos géneros y distintos medios, se convierte ahora en una forma de relato continua y transmedia" (Carrera, Limón, Herrera y Sainz, 2013, p. 537), lo que consecuentemente genera que el relato periodístico deje de ser un producto terminado, listo para su consumo, y pase a ser un sistema integrado de mensajes de distinta naturaleza en continua transformación.

Se ve, por lo tanto, que existe la necesidad de implementar un modelo comunicativo en el que una historia periodística trabajada profesionalmente se expanda a través de diferentes medios, enriqueciéndose con las características particulares de cada soporte, para hacer que el público se involucre con el relato y brinde un aporte valioso a este. Esto es lo que denominamos periodismo transmedia. A continuación, detallaremos los desafíos que el modelo transmedia plantea al periodismo en la actualidad.

\section{La narración de historias periodísticas}

Según Peñafiel (2015), asistimos a un momento clave en el que todo apunta a que la comunicación beberá del concepto transmedia, produciendo mensajes independientes para difundir un mensaje global. La narrativa transmedia es la forma en que la historia reclama sus derechos, principalmente el de expandirse (Rodríguez, 2014). Uno de los principales retos que el escenario transmedia presenta al periodismo es la elaboración, no de noticias, sino de historias periodísticas, es decir, la creación de mundos narrativos complejos que no agoten su contenido en un solo medio o formato. Se busca que el relato periodístico discurra entre la diversidad de canales que ahora existe, creciendo y enriqueciéndose con cada nuevo punto de acceso.

En concreto, ¿cómo puede el periodismo valerse de una estrategia transmedia? Por un lado, si bien se generan un gran número de producciones periodísticas a diario y se les difunde a través de diferentes medios y plataformas, en reiteradas ocasiones, muchas de estas tienen que ver con una historia mayor, el reto está en que se les otorgue unidad a todas bajo una macrohistoria y se las articule bajo una lógica transmedia. Ya lo indica Belsunces (2011, p. 30): "los universos transmedia no siempre son concebidos como tal, sino que se expanden a medida que se van desarrollando". Por otro lado, por su naturaleza, existen noticias que desde su nacimiento reclaman un nivel de profundidad mayor que otras, el reto se encuentra aquí en que a partir de estos trabajos se lleguen a configurar universos narrativos con potencial para ser expandidos.

Ahora nos centraremos en el desafío que implica enriquecer las historias periodísticas. La construcción de una historia transmedia pasa por el diseño de un universo amueblado con una geografía detallada, con personajes y sus respectivas psicologías, con una 
historia dotada de hitos decisivos, cronologías: en definitiva, un universo que no deja de evolucionar, aun cuando dejemos de mirarlo (Rodríguez, 2014, p. 31).

Para que esta comunicación transmedia consiga éxito, específicamente en el periodismo, tendrá que demostrar su capacidad para que cada una de sus pequeñas partes informativas dirigidas a cada uno de los medios más idóneos tenga un impacto distinto, enriqueciendo a las anteriores y al total de la comunicación, es decir, aportando valor añadido al esquema comunicativo (Peñafiel, 2015).

No debemos perder de vista que en un terreno competitivo como el de hoy es necesario innovar y crear contenidos diferenciados y relevantes, donde haya espacio para la reflexión y el análisis (Peñafiel, 2015). El reto de crear historias periodísticas va más allá de las formas, recae en impulsar un periodismo especializado, preocupado por ofrecer contenidos que no dejen de lado la calidad, y que no se agoten en un solo canal, sino que se abran paso en diversos medios.

\section{Acercar la información al nuevo ciudadano}

Uno de los principales fines de la narrativa transmedia es acercar la historia al público, aspecto que para el periodismo no debe ser indiferente. Así, señala Barrios que:

Hoy el foco de atención está en encontrar esa red de consumidores con intereses comunes que son atraídos por una propuesta comunicativa a la cual ellos contribuyen con sus experiencias e historias, aquello que los usuarios consideran verdaderamente interesante y pueden llegar a compartir con emoción y entusiasmo. (2016, p. 167).

Como señala Scolari (2014), la narrativa transmedia integra dos dimensiones: la construcción de una historia que se expande y la participación activa de los usuarios en este proceso. Se ve que existe una relación de causa-consecuencia entre ambos puntos, pues para lograr que la audiencia se involucre se debe primero haber llegado de la mejor forma a ella.

En el campo periodístico, este acercamiento de las historias al nuevo ciudadano, disperso entre la diversidad de canales del actual ecosistema mediático, constituye ya un desafío para sus profesionales. Para Peñafiel $(2015$, p. 3), todo parte de impulsar un periodismo de calidad "que aporte valor añadido a los usuarios de la comunicación, donde cada día hay millones de informaciones que compiten por una audiencia potencial". Hace falta entonces un replanteamiento en la labor periodística para responder a las necesidades del mercado (Barrios, 2016).

Peñafiel (2015) defiende que son el talento, las habilidades personales e intelectuales de los periodistas, las que ahora más que nunca promoverán el desarrollo del sector de la comunicación transmedia. Por lo tanto, el periodista "debe dominar cada medio y ser competente en el quehacer profesional (...) que exige el surgimiento de nuevos lenguajes 
y el establecimiento de una relación más cercana y de complicidad entre el medio de comunicación y sus usuarios" (Barrios, 2016, p. 164).

\section{Impulsar el contenido generado por el usuario y la participación ciudadana}

La circulación de información ha cambiado y como tal, el concepto de recepción, de manera que "los usuarios (...) pueden ser considerados como otro potencial emisor de mensajes, puesto que pueden producir contenidos para distintos medios de comunicación, redes sociales o plataformas comunicacionales" (Peñafiel, 2015, p. 2). "Las genuinas virtudes transmediáticas nos permiten cruzar la línea del cuestionamiento, la indignación o el escándalo ante los medios para transformarnos en buscadores-creadores de información o en actores políticos" (Rodríguez y Peñamarín, 2014, p. 10).

En esta línea hablamos del contenido generado por el usuario (User Generate Content, UGC). En el caso puntual de la información periodística, el UGC es el resultado de que, una vez establecida la conexión con la historia, la audiencia quiera participar de ella, no solo difundiendo el contenido, sino también generando el suyo. De esta forma, el desafío del periodismo recae en presentar una historia tan atractiva que invite a la audiencia a brindar un aporte valioso que contribuya a la construcción del mundo narrativo.

Cabe aclarar en este punto que la generación de contenido por parte de la audiencia no se trata de un ejercicio periodístico. El periodismo es una profesión y solo puede ser ejercida por quienes han sido formados profesionalmente en él. En una entrevista hecha por Scolari (2013, pp. 191-192) a Orihuela, este señala que el UGC no es periodismo, pero sin serlo es innegable que este "ha transformado el espacio de la comunicación pública, las dinámicas de filtrado de información, los procesos de establecimiento de agenda, y también las funciones sociales y políticas del periodismo y de los medios". De esta forma, agrega Orihuela, el hecho de que la audiencia tenga una voz pública se ha convertido en un nuevo mecanismo distribuido de fiscalización del poder político y también de la actividad periodística.

En todo este apartado se ha estudiado cómo la narrativa transmedia en el ámbito periodístico plantea determinados desafíos para quienes ejercen esta profesión y pone al alcance del público los medios necesarios para una participación activa dentro del escenario social que experimenta. Como hemos visto, todo parte de una historia bien contada, por ello, en el siguiente punto desarrollaremos una serie de estrategias a tener en cuenta para la creación de historias periodísticas transmedia.

\section{La implementación del periodismo transmedia}

La narrativa transmedia seguirá estando estos próximos años en pleno auge, debido al número de medios interactivos y plataformas que han ido surgiendo y, también, gracias a que existe ahora una audiencia participativa que demanda nuevos contenidos 
y que está saturada de espacios repetitivos y poco innovadores (Peñafiel, 2015). En el ámbito periodístico se debe, por lo tanto, preparar el terreno para el desarrollo de historias que respondan a las necesidades actuales, tanto del lado de los profesionales, como del público.

A continuación, presentaremos cinco estrategias para el desarrollo de historias periodísticas basadas en el modelo transmedia.

\section{Elaborar un guion transmedia}

Ossorio (2013, p. 489) concibe a la narrativa transmedia como el modelo de "producción de contenidos que construye un relato global a través de relatos independientes en diferentes formatos, y cuya convergencia aporta una historia única que se complementa con las historias y características de cada una de las partes que forman el todo". Esta idea la vemos también en Scolari (2009) y Belsunces (2011), quienes hablan de una macrohistoria o historia global, conformada por las diversas extensiones del relato transmedia.

En esta construcción de todo un mundo narrativo periodístico' resulta fundamental la figura del periodista como el responsable de crear toda una estructura armónica de contenidos informativos (Porto, 2011; Arrojo, 2015; Lovato, 2015; Barrios, 2016). Como explica Porto, "la forma de hacer contenido transmedia (...) exige más de los autores. No se produce como antes. Existe una necesidad distinta de proyectar, planificar los caminos y los contenidos distintos para, al final, producir" (2011, pp. 32-33).

La labor del periodista requiere definir cuál es el centro del relato y, al mismo tiempo, poseer un adecuado conocimiento de los diversos medios de comunicación y de cómo codificar la propuesta comunicativa en cada uno de ellos, de modo que se brinden herramientas de construcción narrativa que vinculen y conecten las historias proporcionadas a través de otras plataformas² (Barrios, 2016).

Siguiendo esta línea, Porto (2011) habla, precisamente, de la elaboración de un guion que permita visualizar los distintos caminos que seguirían entre sí los contenidos y las relaciones entre ellos. Lovato (2015, p. 36) lo denomina guion transmedia, "un elemento complejo que debe preservar, a lo largo de la producción, la suficiente flexibilidad para incorporar el trabajo expansivo de las audiencias, para reunir y agregar nuevos nodos narrativos, y permitir la interactividad".

Este esquema de comunicación debe tener en cuenta los actores que forman parte del proceso (público, medios, plataformas, tecnología, contexto, ambiente), bajo los cuales se concibe, produce, circula y recibe el mensaje, para, a la par, generar nuevas narrativas que impacten no solo al emisor inicial sino a las redes de receptores y amigos (Barrios, 2016).

Según Ossorio (2013), el mundo es la idea general que se transmite, es decir, la propia historia, conformada a su vez por múltiples elementos tales como personajes, acciones, lugares, etc.

2 Arrojo (2015) explica que es necesario conseguir que cada una de las piezas informativas tenga coherencia por sí misma y, a su vez, forme parte de un conjunto homogéneo que permita ampliar la experiencia de usuario. 
Para una idea más clara del guion transmedia, tenemos el documental argentino Mujeres en venta. Como explica Lovato (2015), para este documental, lanzado entre febrero y diciembre del 2015, se utilizaron diversos canales: un webdoc o documental multimedia interactivo, afiches en vía pública con interacción a través de realidad aumentada, videos para LED de interior y exterior, una serie de cómics impresos y digitales, un documental para televisión, movisodios, spots para televisión y radio, un mapa interactivo y un libro multiplataforma. ¿Cómo se planificó? El webdoc fue el corazón de la macro historia y en conexión con este se desarrollaron las piezas para los otros soportes, las mismas que se ocuparon de ampliar argumentos o abordar temas y casos específicos (Lovato, 2015).

Como se ve, la idea de una planificación del guion transmedia es cuidar y enriquecer la macrohistoria, de manera que la audiencia la asuma así. Rodríguez, Ortíz y Sáez (2014, p. 74) sostienen que cada uno de los medios debe hacer lo que mejor sabe con dicho universo, "sin caer en redundancias, sino expandiéndolo y profundizándolo al mismo tiempo".

\section{Formular narrativas diferenciadas para cada plataforma}

Una vez construida la historia global del hecho, lo que corresponde es crear narrativas diferenciadas según cada medio para así enriquecer el mensaje. Ossorio (2013) explica que se debe diseñar de manera tal la comunicación, que en cada medio el usuario obtenga un mensaje independiente del resto, aunque en relación con la idea global. De manera que, si consume únicamente uno de los mensajes tendrá la suficiente cantidad de información como para comprender lo que se quiere transmitir, pero su experiencia se verá enriquecida si consume los mensajes ofrecidos de varios medios, ya que se complementarán entre sí y aportarán distintas visiones y datos del mensaje global.

En este sentido, para Porto y Flores (2012, p. 83), "es necesario formular simultáneamente narrativas diferenciadas, adaptadas a cada una de las plataformas que se utilicen. Así se conseguirá el acercamiento de los productos periodísticos a unos usuarios potenciales heterogéneos". Lo mismo sugiere Barrios al proponer la estructuración de un eje narrativo para cada plataforma o medio, de manera que "cada una de las partes queda interconectada de algún modo, respetando el principio de la narrativa transmedia, en la que no cabe la linealidad" (2016, p. 171).

Para Ossorio, el objetivo de la narrativa transmedia es "enriquecer los mensajes gracias al uso de varias plataformas, que proporcionan al usuario discursos adaptados a cada medio y que ofrecen parcelas de una misma realidad aprovechando las particularidades de cada formato utilizado" (2013, p. 491). De esta forma, según el autor, se debe tener en cuenta que la narrativa transmedia no publica los mismos datos en cada canal, como muchos medios de comunicación lo hacen en la práctica, sino que elabora un mensaje específico para cada plataforma con el fin de aprovechar las potencialidades que cada una de ellas ofrece. 


\section{Generar contenidos que promuevan el diálogo de la audiencia}

Este nuevo contexto hace inevitable una revisión de la noción de audiencia. Las tecnologías digitales, y particularmente el uso de internet, han contribuido a configurar un nuevo modelo de consumidor de medios, más activo, participativo y autónomo, que, a su vez, ya no puede ser percibido como un bloque homogéneo sino como un colectivo fragmentado (Belsunces, 2011).

A este nuevo público apunta el modelo transmedia. Se busca establecer una conexión entre él y la historia, y entre los mismos miembros que lo conforman, para hablar e intercambiar sobre aspectos del relato. Como indica Peribáñez (2013, p. 31), actualmente "la cooperación entre productores y lectores transforma por completo el proceso productivo tradicional, basado en la dicotomía emisor/receptor".

Belsunces (2011) explica que el público controla mejor el flujo de los medios para interactuar con otros consumidores, dejando atrás su predecibilidad para volverse a la vez una audiencia migratoria y leal a las cadenas, redes y medios, constituyendo grupos y comunidades que expresan públicamente sus deseos y sus críticas, hecho que les otorga un poder nunca antes visto.

En este escenario, ¿cómo promover entonces el diálogo fluido entre y con las audiencias? "La clave está en sorprender con contenidos y narrativas a través de diferentes plataformas, en las que el comunicador se adapta a los nuevos modelos y esquemas con los cuales llegar a la gente" (Barrios, 2016, p. 173).

La participación plena de los usuarios, propia del transmedia, exige de los creadores de la historia periodística una actualización constante de ella, con aportes de nuevo contenido en cada canal que se utilice (Peribáñez, 2013). De esta forma, el papel de la industria es la construcción de un primer marco de contenido (macrohistoria), que, posteriormente, será trabajado por la misma audiencia que lo adecuará a sus gustos y ofrecerá un feedback continuo (Peribáñez, 2013).

Asimismo, Barrios (2016) destaca la creatividad y la originalidad de los contenidos como características importantes para captar la atención del público, pues este, al tener a su disposición un sinnúmero de propuestas similares con las cuales interactuar y satisfacer sus necesidades de información y consumo, buscará constantemente aquel elemento diferenciador.

"La participación de los públicos no es una opción, sino una condición necesaria para que una historia transmedia pueda existir" (Belsunces, 2011, p. 27). Y es que, el papel que juega el receptor-usuario a la hora de expandir el universo narrativo abre un campo de posibilidades muy amplio tanto para los productores como para la propia audiencia (Paíno y Rodríguez, 2016). Esto se debe a que da paso a la creación de contenido, punto que desarrollaremos a continuación. 


\section{Plantear proyectos colaborativos}

Partiendo de que una de las principales características de la narrativa transmedia es la capacidad de implicación del seguidor en la historia, cuando se idea un universo narrativo es necesario también plantearse cuál será la experiencia de la audiencia en cada uno de los medios y el grado de participación que podrá experimentar (Costa y Piñeiro, 2012).

Ya que el periodismo es una de las áreas de la comunicación que busca que el público participe e interactúe, debe ofrecer los medios para estar en contacto con su audiencia, escucharla, responderle y obtener de ella ayuda para la configuración de los mensajes y datos que le servirán para ofrecer productos basados en sus intereses y preferencias, lo cual genera un vínculo entre el medio y el público (Ossorio, 2013).

De esta forma, otra de las tareas pendientes que los periodistas tienen al momento de planificar el modelo comunicativo de un hecho periodístico es incentivar el intercambio recíproco entre ellos y el público. ¿Cómo logarlo? Barrios (2016, p. 165) habla de "un juego de dominio propuesto desde los medios en donde se le da ciertas libertades al usuario para reconstruir los hechos y generar nuevas narrativas desde sus redes sociales y microblogs", a la vez que se aprovecha el conocimiento del público para proponer alternativas, respuestas y soluciones acordes a su contexto.

Fernández señala que "las dinámicas comunicativas del siglo XXI se basan cada vez más en el planteamiento de proyectos colaborativos generados en las redes y los medios sociales" (2014, p. 56). Estas plataformas son los medios más idóneos para lograr esa interactividad entre la historia y el usuario, intercambio que puede muy bien terminar en una serie de contenidos generados por el público mismo.

Esta fórmula, detalla Fernández (2014), permite a los creadores de la historia aprove char el talento de la comunidad online, obteniendo resultados más creativos y, al mismo tiempo, garantizando que la iniciativa obtenga un notable alcance, pues los participantes contribuyen activamente a su difusión al sentirse, justamente, autores del producto final. El investigador agrega que mediante esta sensación de implicación directa se genera un mayor grado de fidelización que favorece la circulación de ideas y la diseminación de la macrohistoria a lo largo de múltiples soportes y plataformas.

\section{Generar nuevas puertas de acceso al relato noticioso}

"Una historia transmedia crea un espectador más activo quien interactúa con más plataformas" (Granitz y Forman, 2015, p. 44). Estas plataformas, como hemos explicado, se convierten en las distintas puertas de acceso a la historia que tiene la audiencia, las cuales incluyen medios tradicionales y online, pero que también dan un espacio para cierto tipo de actividades que promueven el universo narrativo y determinados productos que guardan relación con él.

Tal como señala Barrios (2016, p. 174), en este entorno transmedia, donde se conjuga el papel de emisor y receptor, "todos en un momento determinado se encuentran en una 
u otra posición, enriqueciendo el discurso con la participación conjunta y las particularidades de cada uno". Así, la idea de esta estrategia transmedia es que los productos periodísticos encuentren maneras de manifestarse en espacios que quizá antes podrían resultarles ajenos y que el público no concebía como plataformas para la difusión de información.

Se trata, por lo tanto, de que los periodistas innoven, y de que los distintos canales comunicacionales que ahora existen no se limiten a aquellos que se les considera propiamente medios o plataformas de comunicación, sino que un hecho periodístico también pueda expandirse mediante conferencias de prensa, eventos en pro de una causa benéfica, productos como reportajes en DVD, películas basadas en la historia periodística o ropa con frases en alusión al hecho, por citar ejemplos.

\section{Los millennials y el consumo mediático}

La generación millennial, también conocida como generación Y incluye a la población nacida entre los años 80 e inicios del siglo XXI (Claret, 2015; Raines, 2002). Se trata de una generación de jóvenes sociables, optimistas, talentosos, bien educados, colaboradores y de mente abierta (Raines, 2002). Según Elan, Stratton y Gibson (2007, pp. 21-22), la época en la que nacieron los millennials ha inculcado en ellos una serie de cualidades positivas: "son trabajadores y han sido formados por sus padres para tener éxito en la vida. Se han involucrado en numerosas actividades académicas, extracurriculares y de servicio".

Los millennials se diferencian de sus generaciones predecesoras principalmente por tener un mayor nivel de estudios, ser más seculares y haber nacido en la era de la irrupción de internet en todas las esferas de la vida, por lo que tienen un acceso a la información más rápido y completo del que tuvieron sus padres (Claret, 2015). De hecho, se trata de la primera generación que ha pasado toda su vida en el entorno digital, de manera que las tecnologías de la información y la comunicación (TIC) afectan profundamente el cómo viven (Bolton et al., 2013).

Abordemos con detalle esta generación de jóvenes en el ámbito peruano y su relación con las nuevas tecnologías de la comunicación. En el Perú, siete de cada diez personas que viven en zona urbana utilizan internet más de tres veces por semana; y, en el caso de los jóvenes, la frecuencia de consumo, en este mismo universo, llega a ser diaria (Gfk Perú, 2015). Según el Instituto Nacional de Estadística e Informática (2014) —en su informe Estado de la niñez y adolescencia 2014-el 79,7\% de niñas, niños y adolescentes, utiliza con alta frecuencia el internet como principal fuente de información. Todos estos datos, unidos a la gran penetración de los teléfonos inteligentes en el país, nos presentan un panorama de interés que merece ser estudiado por la academia. Este uso de nuevos medios "está cambiando el mercado, el lugar de trabajo y la sociedad; y en última instancia conducirá a nuevos modelos de negocio, procesos y productos" (Bolton et al., 2013, p. 260). 
En este contexto, resulta necesario recoger información que nos permita entender cómo la internet está afectando la vida de los jóvenes. Si bien existe abundante bibliografía sobre esta temática a nivel nacional e internacional, se echan en falta estudios focalizados en la realidad de los escolares. Por ello, el presente estudio pretende ser un aporte, mostrando, gracias a lo extraído de una investigación más amplia, cómo los escolares piuranos se comportan frente a las posibilidades de la red.

La investigación relacionada con el consumo de medios y la interacción con los entornos digitales ha estado encuadrada normalmente en lo que se denomina media effect o efecto de los medios ${ }^{3}$, en la medida que se aborda cómo los medios influyen en la vida de las personas. En este escenario, la investigación que presentamos aborda una aproximación a un público clásicamente estudiado en otras latitudes, pero que carece de literatura específica para el caso del Perú y sus regiones. Por ello, para aproximarnos a la temática que se abordará, se presenta un panorama de las investigaciones relacionadas con el público escolar y adolescente. Esto nos permitirá valorar adecuadamente los resultados de la investigación con miras a enmarcarlos en un contexto más amplio de estudios que aborden al mismo público que ha sido objeto de la investigación.

Como antecedente reciente de estos estudios en Latinoamérica, tenemos la investigación de López (2003), quien concluyó que los jóvenes pueden pasar más tiempo en relación con los medios que con su propia familia. En esta investigación, López evidenció que el consumo de los medios de comunicación forma parte fundamental de los hábitos culturales de consulta e interrelación de los jóvenes, quienes los utilizan como una herramienta para informarse o entretenerse. Para arribar a esta conclusión, el autor exploró las motivaciones de los jóvenes para consumir los medios, el tipo de medio utilizado, los usos que le daban (especialmente a internet, que por esos años había alcanzado relevancia en el ecosistema mediático), y las preferencias por los contenidos. A partir de todos los datos, esta investigación llamaba a una reflexión sobre la necesidad de una educación de audiencias, debido al gran tiempo que invierten los jóvenes en los medios.

La relación entre consumo de medios y participación ciudadana ha sido estudiada por Arriagada y Schuster (2008) para el caso de los jóvenes chilenos. Los investigadores apuntaban a un nuevo modelo de participación ciudadana, que contempla la presencia en chats y fotologs como medios de interacción. Para Arriagada y Schuster (2008, p. 42), "se debería considerar al consumo de nuevos medios de comunicación no sólo como fuentes de información, sino más bien como un espacio de interacción donde los jóvenes sienten que ejercen su ciudadanía al compartir información con sus pares". En todo caso, los investigadores detectaron que, ni los medios tradicionales ni los nuevos medios fomentaban una participación ciudadana activa en términos tradicionales, por lo que para este público su modo de sentirse parte del grupo social consistía en interactuar a través de los nuevos medios.

Lasswell (1927) es considerado el precursor de la primera fase de los media effects. Pero se puede señalar con propiedad que los estudios de los media effects incluyen un conjunto de teorías que explican cómo los mass media influyen en las actitudes y percepciones de la audiencia (Neuman y Guggenheim, 2011). 
Por su parte, Bringas, Rodríguez y Herrero (2008) realizaron un estudio sobre el rendimiento escolar y el uso de los medios de comunicación, y encontraron que el uso de ordenadores puede incrementar el éxito escolar, en cuanto este equipo está relacionado muchas veces con tareas académicas; incluso sus resultados demuestran que el uso de ordenadores con videojuegos no afecta el aprovechamiento escolar. Los autores encontraron una relación entre el bajo rendimiento académico de los alumnos y la visualización de televisión por la noche; mientras que, si los alumnos ven televisión durante el día, su rendimiento académico no se ve afectado. Otro resultado interesante de Bringas, Rodríguez y Herreros (2008) es esta constante: no es el uso de los medios lo que genera malos resultados académicos, sino el tiempo que se invierte en establecer relaciones con las amistades (en el caso del estudio, esta reflexión parte del uso de internet y de los mensajes de texto o SMS), ya que esto disminuye la dedicación al estudio. En el contexto actual, dominado por las redes sociales, esta consideración puede ser especialmente relevante.

Thompson (2013) encontró que la influencia de la tecnología en el aprendizaje de los nativos digitales es muy variada y compleja y no determinista, es decir, los nativos digitales tienen un nuevo ecosistema de aprendizaje, pero este no ha cambiado sustancialmente por la tecnología. En este sentido, Thompson (2013) precisa que no se puede afirmar que los nativos digitales puedan aprender por sí solos todos los conocimientos disponibles en el entorno digital, ya que hay áreas del conocimiento donde la figura del profesor es fundamental. La formación del criterio para saber seleccionar lo correcto en internet es básica para el posterior desempeño del alumno.

Merece destacarse, también aquí, el estudio reciente de Echevarría y Meyer (2017), quienes señalan que el uso de las nuevas tecnologías y las redes sociales favorece en los jóvenes la formación de comunidades de sentido, subculturas y microsociedades. Los autores buscan acercarse a la relación positiva entre el empleo de las TIC y la participación política del público juvenil mexicano, y encuentran que esta efectivamente se da, pero de manera poco significativa, puesto que "una proporción muy reducida de usuarios consulta contenidos vinculados con lo público o lo político" (Echevarría y Meyer, 2017, p. 46). Además, concluyen que a pesar de que sí hay un mayor interés en participar en actividades políticas de parte de usuarios de internet en comparación con usuarios de otros medios, este es mínimo (Echevarría y Meyer, 2017).

Pero los estudios de consumo de medios en escolares y adolescentes no solo han estado ligados a la educación, al aprendizaje y a la participación ciudadana, también hay investigaciones que exploran la exposición de la sexualidad de este público en internet. En este campo, Bobkowski, Shafer y Ortiz (2016) encontraron que la autopresentación sexual de los jóvenes (de 13 a 15 años) en internet es producto de una relación parcialmente mediada por los contenidos sexuales que proveen los medios y moderada por la extroversión de la persona, además de contemplar el propio concepto que tiene la persona de su sexualidad. Es interesante que esta investigación no considere únicamente como contenido sexual los cuerpos desnudos o parcialmente desnudos, sino que incluya en sus categorías el contenido sexual sutil y sugerido. 
La tendencia actual en investigación en este campo es la relacionada con los nuevos modos de consumo de los dispositivos móviles como smartphones y tablets. El cambio vertiginoso en la tecnología y las novedades han hecho de este objeto de estudio uno muy interesante actualmente. Lo central aquí es cómo usan los jóvenes los nuevos dispositivos y las gratificaciones que obtienen de ellos. Leung y Zhang (2016) prestaron atención al uso de la tablet y encontraron que la relajación, la búsqueda de información, el estatus y la moda, y la gestión del trabajo son razones instrumentales para el uso de la tablet, mientras que la conexión social en cualquier momento y lugar, la amplitud de la pantalla y la facilidad de uso son motivos intrínsecos para su uso.

Por su parte, Veinberg (2015) centró su investigación en la actitud de los nativos digitales hacia las fuentes de noticias. La autora destaca como una conclusión que "los nativos digitales se han enfocado en los medios de comunicación que están disponibles en pantalla [smartphone, tablet o computadora portátil] que permiten el uso del sonido e imágenes en movimiento" (p. 301). Twitter tiene una importancia central para los nativos digitales a la hora que buscan estar informados, y la mayor competencia para las noticias y la información viene de la propia actividad de los jóvenes en otras redes sociales. También en la línea de consumo de noticias, Struckmann y Karnowski explican que "los dispositivos móviles extienden el uso de noticias a una amplia gama de nuevas situaciones, y, por lo tanto, penetran por completo la vida cotidiana de los usuarios" (2016, p. 315). Este mismo estudio encontró que los dispositivos tradicionales (televisión, radio, periódicos) tienen un uso limitado a determinadas situaciones físicas y sociales.

Luego de esta breve revisión de estudios ligados al consumo de medios de los jóvenes, que enmarca la presente investigación en un contexto de interpretación más amplio, presentamos los resultados del estudio sobre consumo mediático de los escolares de $5^{\circ}$ de secundaria de la región Piura, realizado en el 2016 en colegios privados, con la finalidad de detectar los modos en los que el periodismo se puede valer de la narrativa transmedia para alcanzar y generar interacción en la generación millennial.

\section{Metodología}

El presente trabajo utiliza como insumo parte de los resultados obtenidos en la investigación Los escolares piuranos en la era digital: un estudio a partir de la experiencia de los alumnos de $5^{\circ}$ de secundaria de los colegios privados ${ }^{5}$ de la región (2016)", cuyo objetivo es conocer la experiencia de los jóvenes de la región Piura respecto a la navegación en internet. El estudio fue realizado por los profesores Fernando Huamán y Henry Palomino, del Centro de Investigación en Opinión Pública (CIOP) de la Universidad de Piura (Perú).

En el sistema educativo peruano, los alumnos que cursan el $5^{\circ}$ año de educación secundaria, tienen una edad comprendida entre los 15 y 17 años, salvo pocos casos muy particulares.

5 Se debe tener en cuenta que el estudiar bajo un sistema de educación privada no es una variable directamente ligada con el nivel socioeconómico de los alumnos, ya que el monto de la pensión que se paga en estos colegios es muy variado, desde cantidades muy pequeñas a otras muy elevadas. 
Para la realización de esa investigación mayor se empleó la técnica de la encuesta, que consiste en aplicar un cuestionario estructurado mediante entrevista personal. Para calcular la muestra se establecieron como parámetros un nivel de confianza de $95 \%$, un margen de error de 4,29\% y una representatividad de 98,36\%. Así, de una población de 5033 estudiantes de $5^{\circ}$ de secundaria de colegios privados en Piura, se trabajó con un tamaño de muestra de 473 estudiantes ${ }^{6}$. El muestreo se realizó en dos etapas:

1. Muestreo aleatorio estratificado, teniendo como estratos: provincia (las ocho provincias de la región Piura), sexo (hombre y mujer), zona (urbana y rural) y tipo de institución educativa (de varones, de mujeres y mixta). Se determinó la cantidad de elementos de la muestra correspondiente a cada estrato por afijación proporcional.

2. Muestreo aleatorio por conglomerados (colegios) con probabilidad proporcional a la medida de tamaño del conglomerado.

De los resultados recogidos de este estudio sobre el consumo de medios que hacen estos jóvenes, se ha trabajado con aquellos enfocados a nuestra investigación, para así detectar los modos en los que el periodismo se puede valer de la narrativa transmedia para alcanzar y generar interacción en la generación millennial. A continuación, se presentan los principales hallazgos y sus respectivas reflexiones.

\section{Hallazgos y discusión}

En este apartado veremos una selección de aquellos resultados del estudio de campo que se corresponden con los siguientes ítems de nuestra investigación, ya que resultan relevantes para nuestro objetivo:

- Describir los rasgos de comportamiento de los estudiantes frente a las nuevas tecnologías.

- Identificar cuáles son los contenidos más consumidos por los estudiantes que son usuarios de internet.

- Determinar cuáles son los dispositivos utilizados por los alumnos para conectarse a la red.

Al final de cada tema se presenta el análisis de los datos y las reflexiones a las que se llega en relación al ejercicio del periodismo transmedia. A partir de la presentación total de los datos, esperamos que el lector pueda validar el análisis y la interpretación, y pueda, a su vez, aprovechar los datos en el marco que le resulte más adecuado.

\footnotetext{
Calculado bajo el supuesto población finita y máxima dispersión ( $p=q=50 \%)$.
} 


\section{Comportamiento frente a las nuevas tecnologías}

Tabla 1. Frecuencia con la que realizan las siguientes actividades

\begin{tabular}{|l|c|c|c|c|c|c|}
\hline \multicolumn{1}{|c|}{ Actividad } & Siempre & $\begin{array}{c}\text { Casi } \\
\text { siempre }\end{array}$ & A veces & Casi nunca & Nunca & Total \\
\hline $\begin{array}{l}\text { Buscar información para realizar } \\
\text { tareas }\end{array}$ & $38 \%$ & $34 \%$ & $27 \%$ & $1 \%$ & $0 \%$ & $100 \%$ \\
\hline $\begin{array}{l}\text { Buscar información de otros } \\
\text { intereses }\end{array}$ & $20 \%$ & $30 \%$ & $40 \%$ & $7 \%$ & $3 \%$ & $100 \%$ \\
\hline Ingresar a Facebook & $59 \%$ & $24 \%$ & $13 \%$ & $3 \%$ & $1 \%$ & $100 \%$ \\
\hline Ingresar a Twitter & $4 \%$ & $4 \%$ & $9 \%$ & $7 \%$ & $76 \%$ & $100 \%$ \\
\hline Ingresar a Snapchat & $7 \%$ & $8 \%$ & $10 \%$ & $3 \%$ & $72 \%$ & $100 \%$ \\
\hline Ingresar a Whatsapp & $12 \%$ & $13 \%$ & $13 \%$ & $4 \%$ & $58 \%$ & $100 \%$ \\
\hline Ingresar a Instagram & $3 \%$ & $8 \%$ & $25 \%$ & $23 \%$ & $41 \%$ & $100 \%$ \\
\hline Revisar el correo electrónico & $32 \%$ & $34 \%$ & $27 \%$ & $4 \%$ & $3 \%$ & $100 \%$ \\
\hline $\begin{array}{l}\text { Ver videos (series, películas, } \\
\text { novelas, música, etc.) }\end{array}$ & $49 \%$ & $30 \%$ & $16 \%$ & $2 \%$ & $3 \%$ & $100 \%$ \\
\hline $\begin{array}{l}\text { Escuchar música (solo audio/ } \\
\text { streaming/en línea) }\end{array}$ & $9 \%$ & $26 \%$ & $21 \%$ & $35 \%$ & $100 \%$ \\
\hline Jugar en internet & $9 \%$ & $34 \%$ & $13 \%$ & $38 \%$ & $100 \%$ \\
\hline Realizar otra actividad & $9 \%$ & $13 \%$ & $17 \%$ & $100 \%$ \\
\hline
\end{tabular}

Tabla 2. Evalúe si está de acuerdo o no con las siguientes afirmaciones

\begin{tabular}{|l|c|c|c|}
\hline \multicolumn{1}{|c|}{ Afirmación } & $\begin{array}{c}\text { De } \\
\text { acuerdo }\end{array}$ & $\begin{array}{c}\text { En } \\
\text { desacuerdo }\end{array}$ & Total \\
\hline Internet es una herramienta útil y necesaria en mi vida & $92 \%$ & $8 \%$ & $100 \%$ \\
\hline $\begin{array}{l}\text { Necesito revisar Facebook y otras redes sociales por lo menos } \\
\text { una vez al día }\end{array}$ & $74 \%$ & $26 \%$ & $100 \%$ \\
\hline $\begin{array}{l}\text { El tiempo máximo que podría pasar sin conectarme a internet } \\
\text { sería de una semana }\end{array}$ & $49 \%$ & $51 \%$ & $100 \%$ \\
\hline $\begin{array}{l}\text { Si mis padres me prohibieran usar internet me sentiría ansioso } \\
\text { y desesperado }\end{array}$ & $28 \%$ & $72 \%$ & $100 \%$ \\
\hline Ahora es normal dormir poco por navegar en internet & $48 \%$ & $52 \%$ & $100 \%$ \\
\hline
\end{tabular}


A pesar de que la tendencia señala a Twitter como la plataforma idónea para la difusión de noticias ${ }^{7}$, en el caso de los escolares piuranos, esta red social tiene un uso extremadamente limitado (8\%), mientras que Facebook se presenta como la más adecuada para alcanzar a este público ya que un $99 \%$ la tiene y un $83 \%$ la usa con frecuencia. Hay que potenciar los contenidos audiovisuales porque este público tiende a ver videos en internet, el $66 \%$ lo hace con regularidad y un $27 \%$ eventualmente.

Considerando también que el 74\% de los estudiantes piuranos reconocen la necesidad de estar conectados a las redes sociales, es importante pensar contenido propio para estas plataformas y no solo utilizarlas como ventanas de llamado. Ya señalaban Porto y Flores (2012) lo valioso que es crear narrativas diferenciadas para cada plataforma en busca de acercar la información periodística a usuarios potenciales heterogéneos. Con un contenido pensado en el lenguaje de las redes sociales se puede alcanzar a este público y despertar su interés por los productos periodísticos.

La generación millennial, en concreto los adolescentes de $5^{\circ}$ de secundaria de Piura, le otorgan gran valor a internet; el 92\% considera que es una herramienta útil y necesaria en su vida. Esto refuerza la idea de que el periodismo transmedia debe procurar más presencia en el entorno digital y desarrollar en estos canales contenido amplio sobre temas, por ejemplo, políticos o culturales. Si no se da presencia a las informaciones de este carácter en la versión digital, la generación millennial tendrá realmente poco contacto con estos contenidos vitales para su desarrollo como ciudadanos responsables.

\section{Consumo de contenidos en internet}

Tabla 3. ¿Qué tipo de información consumes mientras navegas en Internet?

\begin{tabular}{|l|c|c|c|}
\hline \multicolumn{1}{|c|}{ Tipo de información } & Sí & No & Total \\
\hline Noticias & $68 \%$ & $32 \%$ & $100 \%$ \\
\hline Espectáculos & $39 \%$ & $61 \%$ & $100 \%$ \\
\hline Arte y cultura & $60 \%$ & $40 \%$ & $100 \%$ \\
\hline Nutrición y salud & $54 \%$ & $46 \%$ & $100 \%$ \\
\hline Tecnología & $79 \%$ & $21 \%$ & $100 \%$ \\
\hline Música & $96 \%$ & $4 \%$ & $100 \%$ \\
\hline Moda y belleza & $44 \%$ & $56 \%$ & $100 \%$ \\
\hline Series y películas & $86 \%$ & $14 \%$ & $100 \%$ \\
\hline Otra & $53 \%$ & $47 \%$ & $100 \%$ \\
\hline
\end{tabular}

Twitter es la red social preferida por los profesionales de la información debido a su rapidez y variedad, es usada como mecanismo de viralización de los contenidos (Arrabal-Sánchez y De Aguilera-Moyano, 2016). 
Tabla 4. De los siguientes servicios, ¿̇cuáles usas?

\begin{tabular}{|l|c|c|c|}
\hline \multicolumn{1}{|c|}{ Cuenta de } & Sí uso & No uso & Total \\
\hline Correo electrónico & $42 \%$ & $58 \%$ & $100 \%$ \\
\hline Facebook & $99 \%$ & $1 \%$ & $100 \%$ \\
\hline Instagram & $40 \%$ & $60 \%$ & $100 \%$ \\
\hline Whatsapp & $85 \%$ & $15 \%$ & $100 \%$ \\
\hline Twitter & $19 \%$ & $81 \%$ & $100 \%$ \\
\hline
\end{tabular}

El 68\% de los escolares consume noticias mientras navega en internet. Esto refuerza la idea trabajada en la primera parte de esta investigación sobre la formulación de un guion transmedia para difundir contenidos informativos que, en este caso, para acercarse a este público, debe contemplar a internet como una plataforma clave en su estrategia. Se encontró también que el 96\% de los estudiantes escuchan música mientras navegan por internet, por esto consideramos que se puede optar con el recurso del podcast de noticias, de tal manera que el público escuche la noticia mientras realiza otras actividades a la par.

Asimismo, las series y películas tienen un alto nivel de consumo (86\%), por lo que se podría destacar que el público joven millennial piurano tiende a buscar historias en internet. En este escenario, repensar el storytelling periodístico para la narración en internet podría dar luces sobre cómo presentar los contenidos noticiosos. Por otro lado, habría que reconducir los esfuerzos de los boletines a través del correo electrónico, ya que un 58\% afirma que no usa este servicio. Por el contrario, el 99\% de los escolares usa Facebook y el $85 \%$ Whatsapp. Con lo cual se confirma la tendencia que desarrollábamos en puntos anteriores de que uno de los puntos clave del ecosistema de medios de los millennials son las redes sociales (Echevarría y Meyer, 2017; Veinberg, 2015).

\section{Conectividad en las plataformas digitales}

Tabla 5. De los siguientes aparatos tecnológicos, ¿cuál tienes?

\begin{tabular}{|l|c|c|c|}
\hline \multicolumn{1}{|c|}{ Aparato tecnológico } & Sí tengo & No tengo & Total \\
\hline Celular sin conexión a internet & $14 \%$ & $86 \%$ & $100 \%$ \\
\hline Celular Smartphone/iPhone & $81 \%$ & $19 \%$ & $100 \%$ \\
\hline Tablet /iPad & $47 \%$ & $53 \%$ & $100 \%$ \\
\hline PC de escritorio & $71 \%$ & $29 \%$ & $100 \%$ \\
\hline Laptop & $61 \%$ & $39 \%$ & $100 \%$ \\
\hline Otro aparato & $33 \%$ & $67 \%$ & $100 \%$ \\
\hline
\end{tabular}


Tabla 6. Cuando tienes que acceder a internet, ¿qué medio utilizas para conectarte?

\begin{tabular}{|l|c|c|c|c|c|c|}
\hline \multicolumn{1}{|c|}{ Medio de conexión } & Siempre & $\begin{array}{c}\text { Casi siem- } \\
\text { pre }\end{array}$ & A veces & Casi nunca & Nunca & Total \\
\hline Celular & $51 \%$ & $25 \%$ & $15 \%$ & $3 \%$ & $6 \%$ & $100 \%$ \\
\hline Tablet /iPad & $2 \%$ & $9 \%$ & $21 \%$ & $17 \%$ & $51 \%$ & $100 \%$ \\
\hline PC de casa & $14 \%$ & $14 \%$ & $27 \%$ & $13 \%$ & $32 \%$ & $100 \%$ \\
\hline PC de casa de familiares & $2 \%$ & $4 \%$ & $22 \%$ & $19 \%$ & $53 \%$ & $100 \%$ \\
\hline Laptop & $19 \%$ & $18 \%$ & $21 \%$ & $9 \%$ & $33 \%$ & $100 \%$ \\
\hline
\end{tabular}

Tabla 7. ¿Qué medio utilizas con mayor frecuencia para interactuar con tus amigos?

\begin{tabular}{|c|c|c|c|c|c|c|}
\hline Red social & Facebook & Snapchat & Whatsapp & Instagram & Twitter & Total \\
\hline Porcentaje & $59 \%$ & $0 \%$ & $41 \%$ & $0 \%$ & $0 \%$ & $100 \%$ \\
\hline
\end{tabular}

Como se vio en la primera parte de este trabajo, se debe pensar en contenidos que llamen a la interacción y al diálogo del público entre sí (Barrios, 2016; Peribáñez, 2013), esto es, que sirvan para sus actividades frecuentes de chat. En este sentido, al generar contenido pensando en la interacción del público con él, hay que considerar como principales plataformas a Facebook (59\%) y a Whatsapp (41\%), que son las más utilizadas por los jóvenes para dialogar entre ellos.

La mayoría de estudiantes cuentan con equipos que les permiten la conexión a internet, destaca que el $81 \%$ tenga un smartphone con conexión a internet. Esto plantea la necesidad de repensar contenidos para plataformas móviles y potenciar la estrategia mobile del periodismo. También hay que pensar en personalizar el contenido ya que de los dispositivos que tienen el smartphone (81\%), la tablet (47\%) y la laptop (61\%) son de uso personal y se puede ofrecer noticias específicas según las preferencias reconocidas a través de la huella digital de los usuarios. Esto se refuerza con la idea de que los estudiantes se conectan a internet básicamente a través del smartphone, ya que un 76\% lo hace con frecuencia a través de este dispositivo móvil. En este contexto surge la oportunidad de crear aplicaciones para ofrecer contenido informativo en este medio, junto con la posibilidad de fomentar el diálogo y la interacción, que es lo que le interesa a este público.

\section{Conclusiones}

La generación de contenidos pensados para el público es una necesidad en el escenario mediático actual. El periodismo se enfrenta a diversas audiencias con características distintas muy marcadas entre sí, lo que implica que el proceso generalista tradicional de producción y difusión de información está ya desfasado y debe ser reemplazado por un 
proceso narrativo que tenga como centro al usuario y que, a su vez, pensando en este, no descuide la calidad de los contenidos.En este sentido, alcanzar a la generación millennial se constituye en un reto para el periodismo. El panorama actual otorga a estos jóvenes una apertura, no solo a nuevas ideas, sino también a otras narrativas, lo que resulta interesante para los medios de comunicación y les supone también un estímulo para el cambio. Las características particulares de los millennials, en específico, aquellas relacionadas al uso de nuevas tecnologías y el consumo que hacen de los medios de comunicación, en mayor proporción de los digitales, proponen a los actuales profesionales de la información un replanteamiento en sus formas de trabajo y en la ideación del producto noticioso.

Gracias al estudio podemos ver que los estudiantes de $5^{\circ}$ de secundaria, generación millenial, de Piura realizan un consumo casi masivo de los medios digitales y que, además, como puerta de entrada a los contenidos ofrecidos por estos, recurre en gran medida a los dispositivos móviles. En este nuevo ecosistema mediático resultan en gran medida necesarias y útiles para los jóvenes las redes sociales, como una fuente de información, interacción y participación.

Siguiendo esta línea, el desarrollo de contenidos informativos debe orientarse al público y ofrecerle historias pensadas específicamente para él tanto en fondo y forma. Si tenemos un público con presencia predominante en internet y redes sociales, las historias periodísticas deben estar también presentes en esos medios. Frente a una audiencia que de manera destacable hace uso de dispositivos móviles, se debe idear contenido que se pueda consumir a través de estas plataformas. Si el perfil del lector/espectador/usuario se hace más audiovisual, la producción de historias periodísticas debe virar también hacia ello.

Ahora bien, hay un punto que es de vital importancia y que en la configuración de un universo narrativo para la expansión de una historia periodística transmedia no debe perderse de vista. Se trata de la calidad de las historias y del trabajo profesional que estas demandan. Si bien las formas y medios para informar cambian y es requisito dominarlas para conectar con el público, el valor social de la profesión es aquello que debe mantenerse intacto. Esto será bien recibido y demandado por el público millennial, que muestra un interés natural por actividades de servicio y privilegian su educación.

Como se debe entender, los profesionales de la información deben ver en esta serie de cambios una oportunidad, pues la implementación de la narrativa transmedia en periodismo está pensada para que todas las partes resulten beneficiadas. Hablamos de historias de contenido relevante e interesante y con presencia en diversos escenarios. Hablamos también de periodistas llamados a la exigencia en su trabajo y que en la variedad de medios que usen sean fieles al fundamento de su profesión. Nos referimos además a empresas periodísticas que innovan y que económicamente pueden ser rentables. Y, por último, hablamos de una sociedad con una participación activa dentro de las historias difundidas. 


\section{Referencias}

Arrabal, G. y De Aguilera, M. (2016). Comunicar en 140 caracteres. Cómo usan Twitter los comunicadores en España. Comunicar, (46), 9-17. doi: 10.3916/C46-2016-01

Arriagada, A. y Schuster, M. (2008). Consumo de medios y participación ciudadana de los jóvenes chilenos. Cuadernos de Información, 22, 34-41.

Arrojo, M. (2015). Los contenidos transmedia y la renovación de formatos periodísticos: la creatividad en el diseño de nuevas propuestas informativas. Palabra Clave, 18(3), 746-787. doi: 10.5294/ pacla.2015.18.3.6

Barrios, A. (2016). Narrativa periodística en la convergencia de medios. Estudios sobre el Mensaje Periodístico, 22(1), 163-176. doi: 10.5209/rev_ESMP.2016.v22.n1.52587

Belsunces, A. (2011). Producción, consumo y prácticas culturales en torno a los nuevos media en la cultura de la convergencia: el caso de Fringe como narración transmedia y lúdica. (Tesis de Máster, Universitat Oberta de Catalunya). Recuperado de http://goo.gl/jcxTMq

Bobkowski, P., Shafer, A. y Ortiz, R. (2016). Sexual intensity of adolescents' online self-presentations: Joint contribution of identity, media consumption, and extraversion. Computers in Human Behavior, 58, 64-74. doi: 10.1016/j.ch6.2015.12.009

Bolton, R., Parasuraman, A., Hoefnagels, A., Migchels, N., Kabadayi, S., Gruber, T., Komarova, Y. y Solnet, D. (2013). Understanding Generation Y and their use of social media: a review and research agenda. Journal of Service Management, 24(3), 245-267. doi: 10.1108/09564231311326987

Bringas, C., Rodríguez, F., y Herrero, F. (2008). Adaptación y motivación escolar: análisis de la influencia del consumo de medios electrónicos de comunicación por adolescentes. Cuadernos de Trabajo Social, 21, 141 153.

Carrera, P., Limón, N., Herrero, E. y Sainz, C. (2013). Transmedialidad y ecosistema digital. Historia y Comunicación Social, 18, 535-545. doi: 10.5209/rev_HICS.2013.v18.44257

Claret, M. (2015). Los millennials egipcios y la "primavera árabe". Movilización social y frustración. Revista de Estudios Internacionales Mediterráneos, (18), 1-43. doi: 10.15366/reim2015.18

Costa, C., y Piñeiro, T. (2012). Nuevas narrativas audiovisuales: multiplataforma, crossmedia y transmedia. El caso de Águila Roja (RTVE). ICONO 14, 10(2), 102-125. doi: 10.7195/ri14.v10i2.156

Darío, R., y Astudillo, F. (2005). Periodismo por dentro. Una pausa en medio del vértigo. Quito: Quipus, CIESPAL.

Echevarría, M., E Meyer, J. (2017). Internet y socialización política. Consecuencias en la participación juvenil. Anagramas, Rumbos y Sentidos de la Comunicación, 15(30), 29-49. doi: 10.22395/angr.v15n30al

Elan, C., Stratton, T., y Gibson, D. (2007). Welcoming a New Generation to College: The Millennial Students. Journal of College Admission, 195, 20-25.

Fernández, C. (2014). Prácticas transmedia en la era del prosumidor: hacia una definición del contenido generado por el usuario (CGU). CIC Cuadernos de Información y Comunicación, 19, 53-67. doi: 10.5209/ rev_CIYC.2014.v19.43903

Gfk Perú. (2015). Uso de internet en el Perú. Lima: Gfk Conecta SAC.

Granitz, N. y Forman, H. (2015). Building self-brand connections: Exploring brand stories through a transmedia perspective. Journal of Brand Management, 22(1), 38-59. doi: 10.1057/6m.2015.1 
Instituto Nacional de Estadística e Informática. (2014). Estado de la niñez y adolescencia (Informe Técnico N. ${ }^{\circ}$ 2, junio 2014). Lima: INEI.

Jenkins, H. (2008). Convergence culture: la cultura de la convergencia de los medios de comunicación. Barcelona: Paidós.

Kovach, B. y Rosenstiel, T. (2012). Los elementos del periodismo. (A. Diéguez R., Trad.). Madrid: Aguilar.

Lasswell, H. D. (1927). Propaganda technique in the world war. Londres: Paul.

Leung, L. \& Zhang, R. (2016). Predicting tablet use: A study of gratifications-sought, leisure boredom, and multitasking. Telematics and Informatics, 33, 331-341. doi: 10.1016/j.tele.2015.08.013

López, D. (2003). El consumo crítico de los medios de la juventud y el lenguaje de la discreción como propuesta pedagógica. Palabra Clave, (9), 2-16.

Lovato, A. (2015). Del periodismo multimedia al periodismo transmedia. En F. Irigaray y A. Lovato (Eds.), Producciones transmedia de no ficción. Análisis, experiencias y tecnologías (pp. 33-52). Rosario: UNR Editora. Recuperado de http://goo.gl/DNjctM

Martínez, T. (2002). Periodismo y narración: desafíos para el siglo XXI. Cuadernos de Literatura, 8(15), 115-123. Recuperado de http://goo.gl/Xp2E0L

Moloney, K. (2011). Porting transmedia storytelling to journalism. (Tesis de máster, Universidad de Denver). Denver. Recuperado de http://goo.gl/P8MGH

Neuman, W. R. y Guggenheim, L. (2011). The evolution of media effects theory: A six-stage model of cumulative research. Communication Theory, 21(2), 169-196.

Orihuela, J. (2011). 80 claves sobre el futuro del periodismo. Madrid, España: Anaya.

Ossorio, M. (2013). Posibilidades de la narrativa transmedia aplicada al periodismo. En F. Ortega y L. Cardeñosa (Eds.), Las media enterprises y las industrias culturales. Investigar la comunicación y los nuevos medios. Libro de actas del III Congreso Internacional Comunicación 3.0 (pp. 488-497). Universidad de Salamanca. Recuperado de https://goo.gl/2OxZtn

Paíno, A. y Rodríguez, M. (2016): Renovarse o morir. Los 'docugames', una nueva estrategia transmedia que reinventa las formas de transmitir la realidad. ICONO 14, 14(1), 155-180. doi: 10.7195/ril4.v14i1.908

Peñafiel, C. (2015). La comunicación transmedia en el campo del periodismo. Supervivencia en el ecosistema digital. Telos: Cuadernos de Comunicación e Innovación, (100), 84-87. Recuperado de https:// telos.fundaciontelefonica.com/archivo/numero 100/supervivencia-en-el-ecosistema-digital/

Peribáñez, S. (2013). Narrativas transmedia en el periodismo: el juego y el videojuego. (Tesis de pregrado, Universidad de Zaragoza). Recuperado de https://goo.gl/jPCHDO

Porto, D. (201 1). Periodismo, redes sociales y transmediación. Razón y Palabra, (78), 27-36. Recuperado de $h t t p: / / g 00 . g l / m 5 h U w v$

Porto, D. y Flores, J. (2012). Periodismo transmedia. Madrid: Fragua.

Raines, C. (2003). Managing millennials. Connecting generations: The sourcebook for a new workplace. Seattle: Crisp Publications.

Robledo, K., Atarama, T. y Palomino, H. (2017). De la comunicación multimedia a la comunicación transmedia: una revisión teórica sobre las actuales narrativas periodísticas. Estudios sobre el Mensaje Periodístico, 23(1), 223-240. doi: 10.5209/ESMP.55593 
Rodríguez, R. (2014). El relato por otros medios: ¿un giro transmediático? Cuadernos de Información y Comunicación, 19, 19-37. doi: 10.5209/rev_CIYC.2014.v19.43901

Rodríguez, R., Ortiz, F. y Sáez V. (2014). Contenidos transmedia de las teleseries españolas: clasificación, análisis y panorama en 2013. Comunication E Society 27(4), 73-94. Recuperado de http://goo.gl/WBWao0

Rodríguez, R. y Peñamarín, C. (2014). Narraciones transmedia y construcción de los asuntos públicos. CIC Cuadernos de Información y Comunicación, 19, 9-16. Recuperado de http://goo.gl/Am66ao

Scolari, C. (2009). Transmedia storytelling: Implicit consumers, narrative worlds, and branding in contemporary media production. International Journal of Communication, 3, 586-606. Recuperado de http://goo.gl/H5fbDd

Scolari, C. (2013). Narrativas transmedia: cuando todos los medios cuentan. Barcelona: Deusto.

Scolari, C. (2014). Don Quixote of La Mancha: Transmedia storytelling in the grey zone. International Journal of Communication, 8, 2382-2405. Recuperado de https://goo.gl/6afHT7

Struckmann, S. y Karnowski, V. (2016). News consumption in a changing media ecology: An MESMstudy on mobile news. Telematics and Informatics, 33, 309-319. doi: 10.1016/j.tele.2015.08.012

Thompson, P. (2013). The digital natives as learners: Technology use patterns and approaches to learning. Computers \& Education, 65, 12-33. doi: 10.1016/j.compedu.2012.12.022

Veinberg, S. (2015). Digital native's attitude towards news sources. Public Relations Review, 41, 299-301. doi: 10.1016/j.pubrev.2014.11.004 